\title{
High Prevalence of Toxoplasma gondii Infection in Miners: A Case-Control Study in Rural Durango, Mexico
}

\author{
Cosme Alvarado-Esquivele, ${ }^{\mathrm{a}, \mathrm{f}}$, Sandy Janet Pacheco-Vega ${ }^{\mathrm{b}}$, Jesus Hernandez-Tinoco ${ }^{\mathrm{c}}$, \\ Luis Omar Berumen-Segovia ${ }^{a}$, Luis Francisco Sanchez-Anguiano ${ }^{c}$, Sergio Estrada-Martinez ${ }^{c}$, \\ Ada Agustina Sandoval-Carrilloc ${ }^{c}$ Jose Manuel Salas-Pacheco ${ }^{c}$, Oliver Liesenfeld ${ }^{d, ~ e}$, \\ Elizabeth Irasema Antuna-Salcido ${ }^{c}$
}

\begin{abstract}
Background: Very little is known about the seroepidemiology of infection with the parasite Toxoplasma gondii (T. gondii) in miners. We determine the association of $T$. gondii infection and the occupation of miner, and the association of seropositivity for $T$. gondii with the socio-demographic, clinical, work and behavioral characteristics of the miners.

Methods: Through a case-control study, 125 miners working in Durango State, Mexico and 250 age- and gender-matched non-miner subjects were examined for the presence of anti-T. gondii IgG and IgM antibodies using enzyme-linked immunoassays. In addition, the presence of $T$. gondii DNA in miners was determined using polymerase chain reaction. Bivariate and multivariate analyses were used to determine the association of socio-demographic, work, clinical and behavioral characteristics of miners with $T$. gondii infection.
\end{abstract}

Results: Anti-T. gondii IgG antibodies were detected in $75(60.0 \%)$ of 125 miners and in $55(22.0 \%)$ of 250 controls (odds ratio $(O R)=5.31$; 95\% confidence interval $(\mathrm{CI}): 3.33-8.47$; $\mathrm{P}<0.001)$. Among IgG seropositive subjects, the frequency of anti- $T$. gondii IgM antibodies was significantly higher in miners $(39 / 75,52 \%)$ than in controls $(8 / 55,14.5 \%)(\mathrm{P}<0.001)$. All T. gondii seropositive miners referred themselves as healthy. Multivariate analysis of socio-demographic, housing, and behavioral characteristics of miners showed that $T$. gon-

Manuscript accepted for publication October 14, 2016

aBiomedical Research Laboratory, Faculty of Medicine and Nutrition, Juarez University of Durango State, Avenida Universidad S/N, 34000 Durango, Mexico

bSecretaria de Salud, Avenida Cuauhtemoc 225 norte, 34000 Durango, Dgo, Mexico

'Institute for Scientific Research "Dr. Roberto Rivera-Damm", Juarez University of Durango State, Avenida Universidad S/N, 34000 Durango, Mexico ${ }^{\mathrm{d} I n s t i t u t e}$ for Microbiology and Hygiene, Campus Benjamin Franklin, Charite Medical School, Hindenburgdamm 27, D-12203 Berlin, Germany

${ }^{e}$ Current address: Medical and Scientific Affairs, Roche Molecular Systems, Pleasanton, CA 94588, USA

fCorresponding Author: Cosme Alvarado-Esquivel, Laboratorio de Investigacion Biomedica, Facultad de Medicina y Nutricion, Avenida Universidad S/N, 34000 Durango, Dgo, Mexico. Email: alvaradocosme@yahoo.com

doi: http://dx.doi.org/10.14740/jocmr2789w dii seropositivity was positively associated with being born in Durango State $(\mathrm{OR}=3.44 ; 95 \% \mathrm{CI}: 1.09-10.7 ; \mathrm{P}=0.03)$, consumption of boar meat $(\mathrm{OR}=5.53 ; 95 \% \mathrm{CI}: 1.49-20.3 ; \mathrm{P}=0.01)$, living in an overcrowded home $(\mathrm{OR}=5.83 ; 95 \% \mathrm{CI}: 1.49-22.8 ; \mathrm{P}=0.01)$, and was negatively associated with cleaning cat excrement $(\mathrm{OR}=0.33$; $95 \%$ CI: $0.11-0.90 ; \mathrm{P}=0.03)$ and consuming goat meat $(\mathrm{OR}=0.16$; 95\% CI: $0.03-0.76 ; \mathrm{P}=0.02$ ).

Conclusions: Surprisingly, our results indicate that miners represent a risk group for $T$. gondii infection. This is the first age- and gendermatched case-control study on the association of $T$. gondii infection and the occupation of miner. Further studies to identify the exact cause of high seropositivity in miners in rural Durango are needed.

Keywords: Toxoplasma gondii; Infection; Seroprevalence; Miners; Case-control study; Epidemiology; Mexico

\section{Introduction}

Infections with the parasite Toxoplasma gondii ( $T$. gondii) are common in humans around the world [1]. These infections may lead to toxoplasmosis characterized by lymph node enlargement, chorioretinitis, or neuropsychiatric manifestations $[2,3]$. Immunocompromised subjects infected with $T$. gondii may develop severe to life-threatening symptoms, most often toxoplasmic encephalitis [4]. In addition, a primary infection with $T$. gondii in pregnant women may lead to fetal infection and congenital toxoplasmosis $[2,5]$. Infection with $T$. gondii is typically acquired by ingestion of raw or undercooked meat containing viable tissue cysts [6], or by ingestion of food or water contaminated with oocysts shed by cats [7]. Other routes of $T$. gondii infection are thought to be rare including organ transplantation [8] and blood transfusion [9].

The epidemiology of $T$. gondii infection in miners has been scantly studied, and we are not aware of any study of this infection in miners in Mexico. The epidemiological link between miners and $T$. gondii may be the close contact with soil and water that could be contaminated with oocyst shed by cats or other felids. In addition, miners work in rural areas where hunting of wild animals is common, and the risk for acquiring infection by eating raw or undercooked meat from $T$. gondii-infected animals is high. The seroprevalence of infection with $T$. gondii and its 
association with risk factors for infection in miners in Mexico are largely unknown. Therefore, we sought to determine the seroprevalence of $T$. gondii infection in miners in a municipality in rural Durango, Mexico, and to determine the association of seropositivity for $T$. gondii with the socio-demographic, clinical, work and behavioral characteristics of the miners.

\section{Materials and Methods}

\section{Study design and population groups studied}

We performed a case-control seroprevalence study of $125 \mathrm{~min}$ ers (cases) and 250 age- and gender-matched non-miner subjects (controls). Cases and controls were examined for the presence of anti-T. gondii IgG and IgM antibodies. Miners were enrolled from December 2015 to August 2016 in a mine located in the San Dimas Municipality, in the northern Mexican state of Durango. Inclusion criteria for the miners were as follows: 1) working in the mine for at least 3 months, 2) 18 years and older, and 3) willing to participate in the study. All cases included in the study were males and had been working from 3 months to 47 years (mean: $11.4 \pm 9.5$ years) as miners. They were $20-87$ (mean: $43.8 \pm 14.6$ ) years old. Controls were subjects randomly selected from the general population in rural Durango [10]. Controls were matched with cases by gender and age. Controls were males aged $20-87$ (mean: $43.85 \pm 14.5$ ) years old and their age did not differ from that in cases $(\mathrm{P}=0.99)$.

\section{Socio-demographic, housing, clinical, work, and behavio- ral data of miners}

Socio-demographic, clinical, work, and behavioral characteristics of the miners were recorded with the aid of a questionnaire. Socio-demographic items included age, gender, birthplace, residence, and socioeconomic level. Housing conditions items included availability of potable water, form of elimination of excretes, years of education of the head of the family, type of flooring at home, and crowding. Assessment of crowding was performed by dividing the number of people by the number of bedrooms in a house. "Semi-crowed" was considered when 1.6 - 3.5 people were living in a single bedroom. "Overcrowded" was considered when 3.6 or more people were living in a single room. The socioeconomic level of participants was selfreported. A "low socioeconomic status" was considered when a participant lived in poverty, whereas a "medium socioeconomic status" was considered when a participant did not live in poverty or wealth but in an intermediate status.

Clinical data included presence of any illness, history of lymphadenopathy, surgery, transplant, or blood transfusion, impairments in memory, reflexes, hearing and vision, frequent abdominal pain or headache, and dizziness. We recorded the duration of the activity as a miner for each participant. Behavioral items were contact with cats or other animals, cleaning cat feces, foreign traveling, type of meat consumed, frequency of meat consumption, eating raw or undercooked meat, animal brains, beef liver, and dried or cured meat, untreated water or unpasteurized milk, and unwashed raw vegetables or fruits. In addition, behavioral items included washing hands before eating, frequency of eating out of home (in restaurants or fast food outlets), alcoholism, tobacco smoking, and soil contact.

\section{Detection of T. gondii IgG and IgM antibodies}

Serum samples of miners were analyzed for anti-T. gondii IgG antibodies with the commercially available enzyme immunoassay kit "Toxoplasma IgG" (Diagnostic Automation Inc., Woodland Hills, CA, USA). Levels of anti-T. gondii IgG antibody were expressed as International Units (IU)/mL, and a result $\geq 8 \mathrm{IU} / \mathrm{mL}$ was considered positive. Serum samples with anti-T. gondii $\operatorname{IgG}$ antibodies were further tested for anti- $T$. gondii IgM antibodies by the commercially available enzyme immunoassay "Toxoplasma IgM" kit (Diagnostic Automation Inc.). All assays were performed according to the manufacturer's instructions. Positive and negative controls included in the kits were included in each run.

\section{Extraction of DNA and detection of T. gondii DNA}

Miners with Toxoplasma-specific IgG antibodies by EIA were further examined for $T$. gondii DNA by nested-polymerase chain reaction (PCR). Extraction of DNA from whole blood was performed following a protocol described by Iranpour and Esmailizadeh (http:/www.protocol-online.org/prot/Protocols/ Rapid-Extraction-of-High-Quality-DNA-from-Whole-BloodStored-at-4-C-for-Long-Period-4175.html). PCR amplification was performed with primers directed against the $\mathrm{B} 1$ gene of $T$. gondii and following the protocol described by Roth et al [11]. Amplification products were analyzed with $2 \%$ agarose gel electrophoresis, stained with ethidium bromide, and visualized by ultraviolet transillumination.

\section{Statistical analysis}

Results were analyzed with the aid of the software Microsoft Excel 2010, Epi Info version 7 (Centers for Disease Control and Prevention: http://wwwn.cdc.gov/epiinfo/) and SPSS version 15.0 (SPSS Inc. Chicago, IL). For calculation of the sample size, we used a $95 \%$ confidence level, a power of $80 \%$, a $1: 2$ proportion of cases and controls, a reference seroprevalence of $23.8 \%$ [10] as the expected frequency of exposure in controls, and an odds ratio (OR) of 2 . The result of the sample size calculation was 115 cases and 229 controls. We compared the age of cases and controls using the Student's $t$-test. The Pearson's Chi-square test and the Fisher exact test (when values were small) were used to determine the association of $T$. gondii seropositivity with the characteristics of miners. As a strategy for multivariate analysis, only characteristics of miners with a $\mathrm{P}$ value $\leq 0.10$ obtained in the bivariate analysis were included in the analysis. OR and $95 \%$ confidence interval (CI) were calculated using logistic regression analysis with the Enter method. A P value $<0.05$ was considered as statistically 
Table 1. Socio-Demographic and Housing Characteristics of Miners and Prevalence of T. gondii Infection

\begin{tabular}{|c|c|c|c|c|}
\hline \multirow{2}{*}{ Characteristics } & \multirow{2}{*}{ Total subjects } & \multicolumn{2}{|c|}{ Prevalence of $T$. gondii infection } & \multirow{2}{*}{ P value } \\
\hline & & No. & $\%$ & \\
\hline \multicolumn{5}{|l|}{ Age groups (years) } \\
\hline 30 or less & 26 & 16 & 61.5 & 0.73 \\
\hline $31-50$ & 59 & 37 & 62.7 & \\
\hline$>50$ & 40 & 22 & 55.0 & \\
\hline \multicolumn{5}{|l|}{ Birth place } \\
\hline Durango State & 98 & 64 & 65.3 & 0.02 \\
\hline Other Mexican State & 27 & 11 & 40.7 & \\
\hline \multicolumn{5}{|l|}{ Educational level } \\
\hline No education & 16 & 9 & 56.3 & 0.72 \\
\hline $1-6$ years & 68 & 42 & 61.8 & \\
\hline $7-12$ years & 36 & 20 & 55.6 & \\
\hline$>12$ years & 5 & 4 & 80.0 & \\
\hline \multicolumn{5}{|l|}{ Socio-economic level } \\
\hline Low & 61 & 42 & 68.9 & 0.04 \\
\hline Medium & 64 & 33 & 51.6 & \\
\hline \multicolumn{5}{|l|}{ Flooring at home } \\
\hline Ceramic or wood & 5 & 3 & 60.0 & 0.87 \\
\hline Concrete & 92 & 54 & 58.7 & \\
\hline Soil & 28 & 18 & 64.3 & \\
\hline \multicolumn{5}{|l|}{ Availability of potable water } \\
\hline In home & 2 & 1 & 50.0 & 0.34 \\
\hline In land & 3 & 3 & 100.0 & \\
\hline In street & 120 & 71 & 59.2 & \\
\hline \multicolumn{5}{|l|}{ Toilet facilities } \\
\hline Sewage pipes & 5 & 3 & 60.0 & 1.00 \\
\hline Latrine or another & 120 & 72 & 60.0 & \\
\hline \multicolumn{5}{|l|}{ Crowding at home } \\
\hline No & 41 & 26 & 63.4 & 0.05 \\
\hline Semi-crowded & 55 & 27 & 49.1 & \\
\hline Overcrowded & 29 & 22 & 75.9 & \\
\hline \multicolumn{5}{|c|}{ Education of the head of family } \\
\hline 7 years or more & 17 & 10 & 58.8 & 0.52 \\
\hline $4-6$ years & 53 & 29 & 54.7 & \\
\hline Up to 3 years & 55 & 36 & 65.5 & \\
\hline
\end{tabular}

significant.

\section{Ethics statement}

The Ethical Committee of the General Hospital of the Secretary of Health in Durango City approved this project. The purpose and procedures of this study were explained to all miners examined. A written informed consent was obtained from each participant.

\section{Results}

Anti-T. gondii IgG antibodies were detected in $75(60.0 \%)$ of 125 miners and in $55(22.0 \%)$ of 250 controls. Seroprevalence of anti- $T$. gondii IgG antibodies was significantly higher in miners than in controls $(\mathrm{OR}=5.31 ; 95 \% \mathrm{CI}$ : $3.33-8.47$; 
Table 2. Bivariate Analysis of Selected Behavioral Characteristics and Infection With T. gondii in Miners

\begin{tabular}{|c|c|c|c|c|}
\hline \multirow{2}{*}{ Characteristics } & \multirow{2}{*}{ No. of subjects tested } & \multicolumn{2}{|c|}{ Prevalence of $T$. gondii infection } & \multirow{2}{*}{ P value } \\
\hline & & No. & $\%$ & \\
\hline \multicolumn{5}{|c|}{ Cleaning cat excrement } \\
\hline Yes & 43 & 21 & 48.8 & 0.06 \\
\hline No & 82 & 54 & 65.9 & \\
\hline \multicolumn{5}{|c|}{ Raising farm animals } \\
\hline Yes & 99 & 61 & 61.6 & 0.47 \\
\hline No & 26 & 14 & 53.8 & \\
\hline \multicolumn{5}{|l|}{ National trips } \\
\hline Yes & 77 & 43 & 55.8 & 0.23 \\
\hline No & 48 & 32 & 66.7 & \\
\hline \multicolumn{5}{|c|}{ Goat meat consumption } \\
\hline Yes & 101 & 56 & 55.4 & 0.03 \\
\hline No & 24 & 19 & 79.2 & \\
\hline \multicolumn{5}{|c|}{ Sheep meat consumption } \\
\hline Yes & 59 & 30 & 50.8 & 0.04 \\
\hline No & 66 & 45 & 68.2 & \\
\hline \multicolumn{5}{|c|}{ Boar meat consumption } \\
\hline Yes & 94 & 61 & 64.9 & 0.05 \\
\hline No & 31 & 14 & 45.2 & \\
\hline \multicolumn{5}{|c|}{ Chicken meat consumption } \\
\hline Yes & 124 & 75 & 60.5 & 0.40 \\
\hline No & 1 & 0 & 0.0 & \\
\hline \multicolumn{5}{|c|}{ Turkey meat consumption } \\
\hline Yes & 65 & 32 & 49.2 & 0.01 \\
\hline No & 60 & 43 & 71.7 & \\
\hline \multicolumn{5}{|c|}{ Pigeon meat consumption } \\
\hline Yes & 72 & 48 & 66.7 & 0.07 \\
\hline No & 53 & 27 & 50.9 & \\
\hline \multicolumn{5}{|c|}{ Quail meat consumption } \\
\hline Yes & 39 & 26 & 66.7 & 0.30 \\
\hline No & 86 & 49 & 57.0 & \\
\hline \multicolumn{5}{|c|}{ Rabbit meat consumption } \\
\hline Yes & 51 & 27 & 52.9 & 0.18 \\
\hline No & 74 & 48 & 64.9 & \\
\hline \multicolumn{5}{|c|}{ Armadillo meat consumption } \\
\hline Yes & 60 & 38 & 63.3 & 0.46 \\
\hline No & 65 & 37 & 56.9 & \\
\hline \multicolumn{5}{|c|}{ Iguana meat consumption } \\
\hline Yes & 24 & 16 & 66.7 & 0.45 \\
\hline No & 101 & 59 & 58.4 & \\
\hline \multicolumn{5}{|c|}{ Badger meat consumption } \\
\hline Yes & 24 & 17 & 70.8 & 0.22 \\
\hline No & 101 & 58 & 57.4 & \\
\hline
\end{tabular}


Table 2. Bivariate Analysis of Selected Behavioral Characteristics and Infection With T. gondii in Miners - (continued)

\begin{tabular}{|c|c|c|c|c|}
\hline \multirow{2}{*}{ Characteristics } & \multirow{2}{*}{ No. of subjects tested } & \multicolumn{2}{|c|}{ Prevalence of $T$. gondii infection } & \multirow{2}{*}{ P value } \\
\hline & & No. & $\%$ & \\
\hline \multicolumn{5}{|l|}{ Degree of meat cooking } \\
\hline Undercooked & 6 & 5 & 83.3 & 0.39 \\
\hline Well done & 117 & 68 & 58.1 & \\
\hline \multicolumn{5}{|l|}{ Raw dried meat } \\
\hline Yes & 85 & 46 & 54.1 & 0.06 \\
\hline No & 39 & 28 & 71.8 & \\
\hline \multicolumn{5}{|l|}{ Chorizo consumption } \\
\hline Yes & 124 & 75 & 60.5 & 0.40 \\
\hline No & 1 & 0 & 0.0 & \\
\hline \multicolumn{5}{|c|}{ Brain of cow consumption } \\
\hline Yes & 48 & 24 & 50.0 & 0.07 \\
\hline No & 77 & 51 & 66.2 & \\
\hline \multicolumn{5}{|l|}{ Unwashed raw vegetables } \\
\hline Yes & 85 & 54 & 63.5 & 0.24 \\
\hline No & 40 & 21 & 52.5 & \\
\hline \multicolumn{5}{|l|}{ Untreated water } \\
\hline Yes & 107 & 66 & 61.7 & 0.34 \\
\hline No & 18 & 9 & 50.0 & \\
\hline \multicolumn{5}{|c|}{ Frequency of eating out of home } \\
\hline Never & 1 & 1 & 100.0 & 0.19 \\
\hline $1-10$ times a year & 84 & 46 & 54.8 & \\
\hline$>10$ times a year & 40 & 28 & 70.0 & \\
\hline \multicolumn{5}{|l|}{ Alcohol consumption } \\
\hline Yes & 67 & 45 & 67.2 & 0.07 \\
\hline No & 58 & 30 & 51.7 & \\
\hline \multicolumn{5}{|l|}{ Tobacco smoking } \\
\hline Yes & 57 & 39 & 68.4 & 0.07 \\
\hline No & 68 & 36 & 52.9 & \\
\hline
\end{tabular}

$\mathrm{P}<0.001)$. Of the 75 anti-T. gondii IgG positive miners, 30 $(40.0 \%)$ had anti-T. gondii IgG antibody levels higher than $150 \mathrm{IU} / \mathrm{mL}, 10(13.3 \%)$ between 100 and $150 \mathrm{IU} / \mathrm{mL}$, and 35 (46.7\%) between 8 and $99 \mathrm{IU} / \mathrm{mL}$. All seropositive controls had $\geq 8 \mathrm{IU} / \mathrm{mL}$ of anti- $T$. gondii $\mathrm{IgG}$ antibodies as determined by the qualitative test. However, we were unable to quantitate further for the specific IgG level in all 55 seropositive controls. IgG levels could be determined in only 36 of 55 seropositive controls; of these 27 (75.0\%) had anti-T. gondii IgG antibody levels higher than $150 \mathrm{IU} / \mathrm{mL}$, two (5.6\%) between 100 and $150 \mathrm{IU} / \mathrm{mL}$, and seven (19.4\%) between 8 and 99 $\mathrm{IU} / \mathrm{mL}$. The frequency of individuals with high $\mathrm{IgG}$ levels was significantly higher in the controls compared to the cases group $(\mathrm{P}=0.003)$.

Of the 75 miners seropositive for anti-T. gondii IgG antibodies, $39(52.0 \%)$ were also positive for anti-T. gondii IgM antibodies compared to only eight $(14.5 \%)$ of the 55 controls seropositive to anti-T. gondii IgG antibodies $(\mathrm{P}<0.001)$. DNA of $T$. gondii was detected in 13 miners, and eight $(61.5 \%)$ of them were positive for anti-T. gondii IgM antibodies.

The frequency of IgG was similar $(\mathrm{P}=0.47)$ in participants working less than 1 year as a miner (62.5\%) and those with 1 - 5 years $(68.8 \%)$ or $>5$ years $(56.5 \%)$; the frequency of IgM was comparable $(\mathrm{P}=0.92)$ in participants with less than 1 year of working as a miner $(25.0 \%)$, those with $1-5$ years $(31.3 \%)$ or those with $>5$ years $(31.8 \%)$.

Concerning socio-demographic and housing characteristics (Table 1), bivariate analysis showed three characteristics potentially $(\mathrm{P}$ values $\leq 0.10)$ associated with $T$. gondii infection: birth place $(P=0.02)$, socioeconomic status $(P=0.04)$, and crowing at home $(\mathrm{P}=0.05)$. Other socio-demographic and housing characteristics of miners including age, educational level, flooring at home, availability of potable water, form of elimination of excretes, and years of education of the head of 
Table 3. Multivariate Analysis of Selected Characteristics of Miners and Their Association With T. gondii Infection

\begin{tabular}{llll}
\hline Characteristics & Odds ratio & $\mathbf{9 5 \%}$ confidence interval & P value \\
\hline Birth place (Durango State) & 3.44 & $1.09-10.7$ & 0.03 \\
Socioeconomic level (low) & 2.05 & $0.73-5.71$ & 0.17 \\
Cleaning cat excrement (yes) & 0.33 & $0.11-0.90$ & 0.03 \\
Goat meat consumption (yes) & 0.16 & $0.03-0.76$ & 0.02 \\
Sheep meat consumption (yes) & 1.10 & $0.34-3.48$ & 0.88 \\
Boar meat consumption (yes) & 5.53 & $1.49-20.3$ & 0.01 \\
Turkey meat consumption (yes) & 0.35 & $0.12-1.00$ & 0.05 \\
Pigeon meat consumption (yes) & 2.20 & $0.80-5.98$ & 0.12 \\
Raw dried meat (yes) & 0.45 & $0.15-1.32$ & 0.15 \\
Brain of cow consumption (yes) & 0.50 & $0.18-1.32$ & 0.16 \\
Alcohol consumption (yes) & 0.83 & $0.27-2.51$ & 0.75 \\
Tobacco use (yes) & 2.40 & $0.85-6.73$ & 0.10 \\
Crowding & & & 0.09 \\
\multicolumn{1}{c}{ Semi-crowded } & 2.78 & $0.86-8.93$ & 0.01 \\
\hline \multicolumn{1}{c}{ Overcrowded } & 5.83 & $1.49-22.8$ & \\
\hline
\end{tabular}

the family had $\mathrm{P}$ values $>0.10$.

With respect to the clinical characteristics, all seropositive miners referred themselves as healthy. The frequency of T. gondii seropositivity was higher in miners without memory impairment $(56 / 85,65.9 \%)$ than in miners with memory impairment $(18 / 39,46.2 \%)(\mathrm{P}=0.03)$. Other clinical characteristics including history of lymphadenopathy, surgery, blood transfusion, impairments in reflexes, hearing and vision, frequent abdominal pain or headache, and dizziness showed no association with seropositivity. None of the miners had received an organ transplant.

Of the behavioral characteristics of the miners examined (Table 2), 10 variables had $\mathrm{P}$ values $\leq 0.10$ in the bivariate analysis: cleaning cat excrement $(P=0.06)$, consumption of meat from goat $(\mathrm{P}=0.03)$, sheep $(\mathrm{P}=0.04)$, boar $(\mathrm{P}=0.05)$, turkey $(\mathrm{P}=0.01)$, and pigeon $(\mathrm{P}=0.07)$, consumption of raw dried meat $(P=0.06)$, cow's brains $(P=0.07)$, alcohol consumption $(\mathrm{P}=0.07)$, and tobacco smoking $(\mathrm{P}=0.07)$.

Multivariate analysis of socio-demographic, housing, and behavioral characteristics of miners with $\mathrm{P}$ values $\leq 0.10$ in the bivariate analysis (Table 3 ) showed that $T$. gondii seropositivity was positively associated with being born in Durango State $(\mathrm{OR}=3.44 ; 95 \% \mathrm{CI}: 1.09-10.7 ; \mathrm{P}=0.03)$, consumption of boar meat $(\mathrm{OR}=5.53 ; 95 \% \mathrm{CI}: 1.49-20.3 ; \mathrm{P}=0.01)$, and overcrowded homes $(\mathrm{OR}=5.83 ; 95 \% \mathrm{CI}$ : $1.49-22.8 ; \mathrm{P}=$ 0.01 ), and seropositivity was negatively associated with cleaning cat excrement $(\mathrm{OR}=0.33 ; 95 \% \mathrm{CI}: 0.11-0.90 ; \mathrm{P}=0.03)$, and consumption of goat meat $(\mathrm{OR}=0.16$; 95\% CI: $0.03-$ $0.76 ; \mathrm{P}=0.02)$.

\section{Discussion}

Very little is known about the epidemiology of $T$. gondii infec- tion in miners. To the best of our knowledge, the correlation of $T$. gondii infection with the occupation of miner has not been assessed by an age- and gender-matched case-control study design. Therefore, we performed an age- and gender-matched case-control study to investigate the association of $T$. gondii infection with the occupation of miner in the northern Mexican State of Durango.

Remarkably, we found that the prevalence of $T$. gondii exposure was significantly higher in miners than in controls. The seroprevalence found in miners in Durango, Mexico is higher than those reported in miners in other countries. A 7.7\% prevalence of infection was found in coal miners in China using the indirect hemagglutination test [12]. In Ukraine, 37.7\% of miners were seropositive for $T$. gondii using complement-fixation, passive hemagglutination, and intradermal toxoplasmin tests [13]. Furthermore, the high seroprevalence of T. gondii exposure found in miners $(60.0 \%)$ is the highest seroprevalence reported in population groups in Durango State so far. Thus, seroprevalence found in miners is higher than the seroprevalences of $T$. gondii infection reported in adults in rural communities in Durango State (23.8\%) [10], in schizophrenic patients $(20 \%)$ [14], in waste pickers $(21.1 \%)$ [15], inmates $(21.1 \%)$ [16], and ethnic groups living in rural communities including Tepehuanos (22.4\%) [17] and Huicholes (33.2\%) [18]. In addition, the seroprevalence found in miners is higher than the weighted mean (19.27\%) national seroprevalence of T. gondii infection found in Mexico [19]. It is not clear why miners had a higher seroprevalence of $T$. gondii exposure than age- and gender-matched controls which were also obtained from rural settings. Seroprevalence of infection with $T$. gondii increases with age as reported in general populations in rural [10] and urban [20] Durango. However, in the present study, no such increase in $T$. gondii exposure with age was observed, and a surprisingly high $(61.5 \%)$ seroprevalence of $T$. gondii infec- 
tion was already observed in the youngest miners aged 18 - 30 years old.

We searched for socio-demographic, work, housing and behavioral characteristics to investigate the high seroprevalence of $T$. gondii in miners. Duration in the activity did not correlate with $T$. gondii exposure. Even miners with less than 1 year as a miners had a high seroprevalence of infection with $T$. gondii. Multivariate analysis showed that $T$. gondii exposure was positively associated with being born in Durango State. This result was unexpected since $T$. gondii exposure has been repeatedly associated with the characteristic of being born out of Durango State in diverse cohorts including the general population in Durango City [20], inmates [16], patients with vision and hearing impairments, cancer, HIV, or undergoing hemodialysis [21], female sex workers [22], elderly people [23], patients with heart diseases [24], and people applying for medical certificates [25]. The association of $T$. gondii infection with being born in Durango State found in this study likely indicates that infection was acquired in Durango State. In fact, traveling did not increase the prevalence of infection with $T$. gondii in miners. Furthermore, multivariate analysis showed that infection with $T$. gondii was associated with consumption of boar meat, and living in an overcrowded home. These characteristics may have contributed to the high seroprevalence of $T$. gondii infection in miners. Consumption of boar meat was associated with $T$. gondii seropositivity in several populations in the region with lower seroprevalence than miners including patients with work accidents [26], elderly people [23], and the general population in Durango City [20]. Concerning the association of infection with $T$. gondii and living in an overcrowded home, this is the first time we found such association in our studies of infection with T. gondii in the region. Living in an overcrowded area has been considered as a contributing factor for infection with $T$. gondii in pregnant women in Nigeria [27]. It is not clear why overcrowding influenced the seroprevalence of $T$. gondii infection in that study, but other factors for infection including poor sanitation and contamination of environment with cat excrement were also present [27]. In addition, in the Third National Health and Nutrition Examination Survey in the USA (1988 - 1994), researchers found that risk for $T$. gondii infection increased in those who lived in crowded conditions [28]. On the other hand, we found that infection with $T$. gondii was negatively associated with cleaning cat excrement, and consumption of goat meat. These factors have been suggested as risks for infection with $T$. gondii exposure by others $[29,30]$.

The present study has some limitations. The sample size is small, and we studied miners working in only one mine. Further studies should include a larger sample size and sample miners of more than one mine. A high frequency $(61.5 \%)$ of positive $T$. gondii PCR assays among miners seropositive for anti-T. gondii IgM antibodies was found. Therefore, further research on the epidemiology of acute cases of $T$. gondii infection in miners should be conducted.

We conclude that miners represent a risk group for infection with $T$. gondii. This is the first age- and gender-matched study on the association of $T$. gondii infection and the occupation of miner. Further studies to confirm our results are needed.

\section{References}

1. Tenter AM, Heckeroth AR, Weiss LM. Toxoplasma gondii: from animals to humans. Int J Parasitol. 2000;30(1213):1217-1258.

2. Montoya JG, Liesenfeld O. Toxoplasmosis. Lancet. 2004;363(9425):1965-1976.

3. Furtado JM, Smith JR, Belfort R, Jr., Gattey D, Winthrop KL. Toxoplasmosis: a global threat. J Glob Infect Dis. 2011;3(3):281-284.

4. Machala L, Kodym P, Maly M, Geleneky M, Beran O, Jilich D. [Toxoplasmosis in immunocompromised patients]. Epidemiol Mikrobiol Imunol. 2015;64(2):59-65.

5. Kravetz J. Congenital toxoplasmosis. BMJ Clin Evid. 2013;2013.

6. Belluco S, Mancin M, Conficoni D, Simonato G, Pietrobelli M, Ricci A. Investigating the Determinants of Toxoplasma gondii Prevalence in Meat: A Systematic Review and Meta-Regression. PLoS One. 2016;11(4):e0153856.

7. Torrey EF, Yolken RH. Toxoplasma oocysts as a public health problem. Trends Parasitol. 2013;29(8):380-384.

8. Filloy A, Garcia-Garcia O, Fernandez-Lorente L. Chorioretinitis as the first sign of acquired toxoplasmosis transmitted from donor following kidney transplantation: case report and review of the literature. Ocul Immunol Inflamm. 2013;21(1):34-35.

9. Foroutan-Rad M, Majidiani H, Dalvand S, Daryani A, Kooti W, Saki J, Hedayati-Rad F, et al. Toxoplasmosis in Blood Donors: A Systematic Review and Meta-Analysis. Transfus Med Rev. 2016;30(3):116-122.

10. Alvarado-Esquivel C, Cruz-Magallanes HM, EsquivelCruz R, Estrada-Martinez S, Rivas-Gonzalez M, Liesenfeld O, Martinez-Garcia SA, et al. Seroepidemiology of Toxoplasma gondii infection in human adults from three rural communities in Durango State, Mexico. J Parasitol. 2008;94(4):811-816.

11. Roth A, Roth B, Hoffken G, Steuber S, Khalifa KI, Janitschke K. Application of the polymerase chain reaction in the diagnosis of pulmonary toxoplasmosis in immunocompromised patients. Eur J Clin Microbiol Infect Dis. 1992;11(12):1177-1181.

12. Zhang GN. [A survey of Toxoplasma gondii infection of miners in the Nantun coal mine]. Zhonghua Liu Xing Bing Xue Za Zhi. 1988;9(2):96-98.

13. Orestenko LP. [Toxoplasmosis in workers in the coal industry]. Zh Mikrobiol Epidemiol Immunobiol. 1972;49(9):131-133.

14. Alvarado-Esquivel C, Urbina-Alvarez JD, EstradaMartinez S, Torres-Castorena A, Molotla-de-Leon G, Liesenfeld O, Dubey JP. Toxoplasma gondii infection and schizophrenia: a case control study in a low Toxoplasma seroprevalence Mexican population. Parasitol Int. 2011;60(2):151-155.

15. Alvarado-Esquivel C, Liesenfeld O, Marquez-Conde JA, Cisneros-Camacho A, Estrada-Martinez S, Martinez-Garcia SA, Gonzalez-Herrera A, et al. Seroepidemiology of infection with Toxoplasma gondii in waste pickers and waste workers in Durango, Mexico. Zoonoses Public 
Health. 2008;55(6):306-312.

16. Alvarado-Esquivel C, Hernandez-Tinoco J, Sanchez-Anguiano LF, Ramos-Nevarez A, Cerrillo-Soto SM, SaenzSoto L, Liesenfeld O. High seroprevalence of Toxoplasma gondii infection in inmates: A case control study in Durango City, Mexico. Eur J Microbiol Immunol (Bp). 2014;4(1):76-82.

17. Alvarado-Esquivel C, Estrada-Martinez S, Garcia-Lopez CR, Rojas-Rivera A, Sifuentes-Alvarez A, Liesenfeld O. Seroepidemiology of Toxoplasma gondii infection in Tepehuanos in Durango, Mexico. Vector Borne Zoonotic Dis. 2012;12(2):138-142.

18. Alvarado-Esquivel C, Pacheco-Vega SJ, HernandezTinoco J, Sanchez-Anguiano LF, Berumen-Segovia LO, Rodriguez-Acevedo FJ, Beristain-Garcia I, et al. Seroprevalence of Toxoplasma gondii infection and associated risk factors in Huicholes in Mexico. Parasit Vectors. 2014;7:301.

19. Galvan-Ramirez Mde L, Troyo R, Roman S, CalvilloSanchez C, Bernal-Redondo R. A systematic review and meta-analysis of Toxoplasma gondii infection among the Mexican population. Parasit Vectors. 2012;5:271.

20. Alvarado-Esquivel C, Estrada-Martinez S, Pizarro-Villalobos H, Arce-Quinones M, Liesenfeld O, Dubey JP. Seroepidemiology of Toxoplasma gondii infection in general population in a northern Mexican city. J Parasitol. 2011;97(1):40-43.

21. Alvarado-Esquivel C, Liesenfeld O, Torres-Castorena A, Estrada-Martinez S, Urbina-Alvarez JD, Ramos-de la Rocha M, Marquez-Conde JA, et al. Seroepidemiology of Toxoplasma gondii infection in patients with vision and hearing impairments, cancer, HIV, or undergoing hemodialysis in Durango, Mexico. J Parasitol. 2010;96(3):505508.

22. Alvarado-Esquivel C, Sanchez-Anguiano LF, Hernandez-Tinoco J, Arreola-Chaidez E, Lopez J, Salcido-Meraz KI, Estrada-Martinez S, et al. High Seroprevalence of Toxoplasma Gondii Infection in Female Sex Workers: A Case-Control Study. Eur J Microbiol Immunol (Bp).
2015;5(4):285-292.

23. Alvarado-Esquivel C, Liesenfeld O, Burciaga-Lopez BD, Ramos-Nevarez A, Estrada-Martinez S, CerrilloSoto SM, Carrete-Ramirez FA, et al. Seroepidemiology of Toxoplasma gondii infection in elderly people in a northern Mexican city. Vector Borne Zoonotic Dis. 2012;12(7):568-574.

24. Alvarado-Esquivel C, Salcedo-Jaquez M, SanchezAnguiano LF, Hernandez-Tinoco J, Rabago-Sanchez E, Beristain-Garcia I, Liesenfeld O, et al. Association Between Toxoplasma gondii Exposure and Heart Disease: A Case-Control Study. J Clin Med Res. 2016;8(5):402409.

25. Alvarado-Esquivel C, Sanchez-Anguiano LF, HernandezTinoco J, Pulido-Montoya RA, Acosta-Rojas G, EstradaMartinez S, Perez-Alamos AR, et al. Seroepidemiology of Toxoplasma Gondii Infection in People Applying for Medical Certificates. Eur J Microbiol Immunol (Bp). 2016;6(2):90-98.

26. Alvarado-Esquivel C, Torres-Castorena A, Liesenfeld O, Estrada-Martinez S, Urbina-Alvarez JD. High seroprevalence of Toxoplasma gondii infection in a subset of Mexican patients with work accidents and low socioeconomic status. Parasit Vectors. 2012;5:13.

27. Onadeko MO, Joynson DH, Payne RA. The prevalence of Toxoplasma infection among pregnant women in Ibadan, Nigeria. J Trop Med Hyg. 1992;95(2):143-145.

28. Jones JL, Kruszon-Moran D, Wilson M, McQuillan G, Navin T, McAuley JB. Toxoplasma gondii infection in the United States: seroprevalence and risk factors. Am J Epidemiol. 2001;154(4):357-365.

29. Guo M, Buchanan RL, Dubey JP, Hill DE, Lambertini E, Ying Y, Gamble HR, et al. Qualitative Assessment for Toxoplasma gondii Exposure Risk Associated with Meat Products in the United States. J Food Prot. 2015;78(12):2207-2219.

30. Jones JL, Parise ME, Fiore AE. Neglected parasitic infections in the United States: toxoplasmosis. Am J Trop Med Hyg. 2014;90(5):794-799. 